\title{
Cambios en el estado afectivo y la atribución emocional durante las fluctuaciones de estradiol del ciclo ovárico en estudiantes universitarias
}

\author{
María Isabel Gómez León \\ Universidad Internacional de la Rioja (España)
}

\begin{abstract}
El objetivo de esta investigación fue analizar si existía algún tipo de relación entre las fluctuaciones de estradiol del ciclo ovárico, dos variables de la afectividad, la distimia y la eutimia, y el tipo de atribuciones emocionales, positivas, negativas o neutras, realizadas a estímulos semánticos y faciales. Para ello se midieron los niveles hormonales de 17- $\beta$ estradiol en saliva, a través de kits ELISA (Enzyme-Linked ImmunoSorbent Assay) en 64 mujeres, de entre 18 y 19 años. Los sujetos realizaron el Inventario de Depresión Estado/Rasgo (IDER) y dos pruebas de atribución emocional, para estímulos verbales y faciales, en dos momentos del ciclo, cuando sus niveles de estradiol eran elevados y cuando éstos eran bajos. Las mujeres estudiadas obtuvieron puntuaciones significativamente superiores en los ítems de eutimia estado y en el número de atribuciones positivas realizadas, tanto a los estímulos faciales como semánticos, cuando sus niveles de estradiol eran mayores, que cuando sus niveles de estradiol eran bajos. Los resultados sugieren que el estradiol podría ser uno de los factores que participan en la modulación y la regulación del estado afectivo positivo. Con esta investigación se pretende dar un nuevo enfoque para reforzar la resiliencia psicológica y promover la salud mental.
\end{abstract}

Palabras Clave: Estado afectivo, atribución emocional, estradiol.

Changes in the affective state and in the emotional attribution during estradiol fluctuations of the ovarian cycle in university students. The purpose of this research was to analyze if some type of relation existed between the fluctuations of estradiol of the ovarian cycle, two variables of the affectivity, the dysthymia and the euthymia, and the type of emotional attributions, positive, negative or neutral, realized to semantic and facial stimuli. The hormonal levels of $17-\beta$ estradiol have measured up in saliva, across kits ELISA (Enzyme-Linked Immuno Sorbent Assay) in 64 women, aged 18-19 years. The subjects realized the Inventory of Depressive State/Characteristic (IDER) and two tests of emotional attribution, for verbal and facial stimuli, in two moments of the cycle, when the levels of estradiol were high and when these were lower. The studied women obtained significantly top punctuations in the items of eutimia state and in the number of positive attributions realized, with both facial and semantic stimuli, when they were during the more high beak of estradiol, that when the levels of estradiol were low. The results suggest that the estradiol might be one of the influential factors in the modulation and regulation of the positive affective state. This research seeks to give a new approach to strengthen psychological resilience and promote mental health.

Keywords: Affective state, emotional attribution, estradiol.

Correspondencia: María Isabel Gómez León. Departamento de Psicobiología. Universidad Internacional de La Rioja. Avda. de la Paz, no 137. C.P.: 26004. Logroño, La Rioja (España). E-mail: mabelgomezleon@gmail.com 
El estado de ánimo negativo constituye actualmente uno de los principales factores de riesgo para contraer enfermedades físicas y mentales (Schwarz, Rief, Radkovsky, Berking, y Kleinstauber, 2017; Shackman, 2016), mientras que el estado de ánimo positivo ha sido asociado con una mayor longevidad (Okely, Weiss, y Gale, 2017; Zhang y Han, 2016).

En general, parece haber un acuerdo en que una persona con un estado de ánimo depresivo tiene una mayor probabilidad de utilizar cogniciones y sesgos negativos en el procesamiento de la información (LeMoult y Gotlib, 2018). Los diferentes estados de ánimo influencian nuestras percepciones y juicios sociales, y, en definitiva, nuestra conducta social, con la considerable repercusión que esto puede tener en la conducta interpersonal, la vida laboral y la salud mental de la persona.

Muchos de estos cambios afectivos han sido asociados a las fluctuaciones hormonales que suceden durante el ciclo menstrual (Del Río, Alliende, Molina, Serrano, Molina, y Vigil, 2018). Una variedad de conductas no reproductivas son sensibles a los esteroides sexuales, y varias áreas cerebrales, no directamente involucradas en la reproducción, contienen concentraciones de neuronas esteroides-sexuales. Tanto el desarrollo de estas áreas como su estructura y función sináptica están moduladas por las hormonas gonadales (Marečková et al., 2014; Pletzer, Kronbichler, Aichhorn, Bergmann, Ladurner, y Kerschbaum, 2010).

La reactividad emocional y el uso y la eficacia de estrategias adecuadas para el control y la regulación emocional varían a lo largo del ciclo menstrual (Armbruster, Grage, Kirschbaum, y Strobel, 2018; Reimers et al., 2014). La capacidad de la mujer de inhibir emociones temerosas varía dependiendo del estado endógeno hormonal, en concreto, la extinción del miedo es menos eficaz en mujeres durante los períodos de estradiol bajos, mientras que el estradiol lo facilita (Li y Graham, 2017) y la memoria de reconocimiento basada en la recuperación de elementos negativos disminuye desde la fase folicular temprana, donde los niveles de estradiol son bajos, al inicio de la fase lútea, donde los niveles de estradiol son elevados (Bayer, Schultz, Gamer, y Sommer, 2014). Tanto la extinción del miedo como la regulación cognitiva emocional dependen de un circuito neuronal común en el que interactúa el córtex prefrontal ventromedial y la amígdala (Schiller y Delgado, 2010), un neurocircuito que está modulado, estructural y funcionalmente, por el estradiol y la progesterona (Goldstein et al., 2005; van Wingen et al., 2008).

En este sentido, una gran parte de la investigación se ha centrado en controlar y disminuir los estados emocionales negativos asociados con los cambios hormonales de la mujer, cuando resultaría igualmente valioso promover estrategias que fomentasen las emociones positivas derivadas o asociadas a ellos (Gordon et al., 2018). Las emociones positivas amplían los recursos físicos, cognitivos y sociales, los hacen más duraderos y aumentan las reservas a las que se puede recurrir cuando se presentan amenazas, además, 
aumentan los patrones para actuar en ciertas situaciones optimizando los recursos personales a nivel físico, psicológico y social (Fredrickson, 2013).

La creciente investigación neurocientífica apoya la opinión de que las emociones positivas no son simplemente lo opuesto a las emociones negativas, sino que pueden ser dimensiones independientes del afecto mental (Bayer et al., 2014; Del Río, Alliende, Molina, Serrano, Molina, y Vigil, 2018).

El elevado nivel de estradiol durante la fase folicular del ciclo menstrual aumenta la sensibilidad hacia las recompensas (Diekhof y Ratnayake, 2016; Dreher et al., 2007; Mulligan et al., 2018) incrementa el tono parasimpático (Armbruster, Grage, Kirschbaum, y Strobel, 2018) y el número de emociones positivas durante el sueño (Natale, Albertazzi, y Cangini, 2003), parece mejorar el humor, el nivel de energía y el bienestar en general (Armbruster et al., 2018; Flöter, Nathorst-Böös, Carlström, y von Schoultz, 2002; Yen et al., 2018) pudiendo favorecer la tendencia a realizar un tipo de atribuciones más positivas, congruentes con el estado de ánimo, durante el procesamiento de la información, tanto verbal como facial, que cuando los niveles de estradiol son bajos.

Este sesgo positivo podría tener beneficios tanto en cuanto a variable influyente en el bienestar psicológico subjetivo, como en cuanto a estrategia para el inicio y el mantenimiento de determinadas terapias cognitivo/conductuales (Gordon et al., 2018).

El objetivo de este estudio es determinar algunos de los efectos del estradiol sobre el estado afectivo y el procesamiento emocional de la mujer durante las fluctuaciones derivadas del ciclo ovárico normal.

\section{MÉTODO}

\section{Participantes}

La muestra fue extraída al azar en el primer curso universitario de una facultad del sur de Madrid.

Todas las alumnas seleccionadas tenían entre 18 y 19.11 años, el total de la muestra fue en un principio de 78 mujeres, ninguna de las cuales estaban tomando, o habían tomado durante los últimos 3 meses, anticonceptivos orales ni estaban recibiendo ningún tipo de tratamiento médico o psicológico. Del total de las alumnas que realizaron las pruebas fueron excluidas posteriormente aquellas que, una vez analizados los niveles de estradiol en saliva, no llegaron a obtener los elevados niveles de estradiol esperados en el día 13 después de la última menstruación, esto es, el primer día de ovulación. De esta forma, la muestra resultante fue de 64 mujeres. 


\section{Instrumentos}

Para el análisis cuantitativo de estradiol se utilizó el método inmunoenzimático de tipo competitivo ELISA (Enzyme-Linked ImmunoSorbent Assay) ya que se trata de un método no invasivo, rápido y de gran sensibilidad (Wang et al., 2015). Las muestras de saliva (3-5 ml) fueron recogidas en Salivette ${ }^{\circledR}$ tubos de esponja (Sarstedt, Nümbrecht, GE). Se utilizaron los kits de prueba de ensayos de inmunoabsorción enzimática Neogen® (ELISA) estradiol, y el sistema de inmunoanálisis multitest Grifols Triturus®.

Para medir el componente afectivo se utilizó el Inventario de Depresión Estado/Rasgo (IDER) (Spielberger, Buela Casal, y Agudelo, 2008), el cual ha mostrado niveles adecuados de confiabilidad y validez en una muestra española. La prueba consta de veinte ítems distribuidos en dos escalas: Rasgo y Estado, cada una con diez ítems, cinco para medir distimia y cinco para medir eutimia. Por distimia y eutimia estado se entiende el grado en el que está presente en el momento de la evaluación la afectividad negativa y positiva, respectivamente. Por su parte, distimia y eutimia rasgo hace alusión a la frecuencia de la presencia de la afectividad negativa y positiva, respectivamente (Spielberger et al., 2008).

Para la presentación de estímulos, tanto semánticos como faciales, se utilizó la interfaz gráfica de E-Prime versión 2.0, con el dispositivo de respuesta Chronos, que permite registrar las respuestas con una precisión de milisegundos.

Los estímulos semánticos constaban de veinticuatro palabras seleccionadas a partir de estudios preliminares (Fernández, Díez, Alonso, y Beato, 2004) en los cuales un amplio grupo de palabras fueron valoradas a través de escalas de agradabilidad que iban del 1 al 7 (punto medio=4), siendo el 1 el menos agradable y el 7 el más agradable. Se seleccionaron cuatro palabras de cada rango considerando su frecuencia léxica, así como número de letras y sílabas. Las letras aparecían en minúsculas, color negro y tipografía Comic Sans MS con un tamaño de 46 puntos.

Las expresiones faciales fueron seleccionadas del Facial Expressions of Emotion-Stimuli and Test (FEEST), elaborado por Young, Perrett, Calder, Sprengelmeyer y Ekman (2002) con la finalidad de evaluar el reconocimiento facial de las seis emociones básicas. Específicamente se utilizó la sección de Rostros continuos transformados, donde la serie de cada emoción está compuesta por 7 imágenes con expresiones emocionales en aumento de intensidad, es decir, $0 \%$ de emoción (neutral), $25 \%$ (transformado), 50\% (transformado), 75\% (transformado), 100\% (prototipo de emoción), $125 \%$ (caricaturizada), $150 \%$ (caricaturizada). Se eliminaron de la serie las imágenes del $125 \%$ y $150 \%$ por ser caricaturizadas, es decir, poco reales. En concreto la serie empezaba por la emoción de tristeza (100\%) hasta la neutral de esta misma emoción, momento en el comenzaba la expresión neutral de felicidad hasta la de felicidad (100\%), suponiendo un total de 10 imágenes. Se presentaron en blanco y negro 
con un tamaño de $424 \times 424$ píxeles, fondo blanco, quedando centradas tanto vertical como horizontalmente.

\section{Procedimiento}

La mitad de las alumnas fue citada el día 13 después de su última menstruación (supuesto pico de estradiol) y 15 días después de este día, la otra mitad fue citada 13 días antes del supuesto pico de estradiol y el día en el que éste supuestamente se produce. En cada encuentro se les recogió una muestra de saliva, que posteriormente fue congelada a $-40^{\circ} \mathrm{C}$, y se les administró la prueba: IDER y atribución emocional, facial y semántica, por este orden, a través del USB Chronos se registró tanto el tiempo de respuesta como la valencia. Tanto la recogida de las muestras en saliva como las pruebas de afectividad y atribución fueron realizadas a la misma hora de la mañana (9:00 h.). Las participantes no podían cepillarse los dientes, beber o comer en los 30 minutos antes de la recogida de saliva.

Para todos los grupos se configuró el programa para mostrar los estímulos, semánticos y faciales, durante $500 \mathrm{~ms}$. El intervalo entre la desaparición del estímulo y la aparición del siguiente (si no se daba una respuesta) fue establecido en $1000 \mathrm{~ms}$, por lo que el tiempo máximo de respuesta era de $1.500 \mathrm{~ms}$, que empezaban a contar desde el mismo momento en el que se iniciaba la presentación del estímulo. El software desarrollado para la presentación de los estímulos y la recogida de datos aleatorizaba el orden de presentación intersujetos pero conservaba el orden de presentación de las secuencias intrasujetos.

Todos los participantes dieron su consentimiento informado en la investigación, cumpliendo con los estándares de seguridad y ética de las leyes del país en que se realizó y siguiendo las directrices éticas de la Declaración de Helsinki.

\section{Análisis de datos}

Los datos fueron agrupados según la variable Estradiol. Las 64 mujeres que realizaron las pruebas durante el pico de estradiol (480-940 pg/ml), fueron denominadas grupo Alto estradiol, estas mismas mujeres fueron denominadas grupo Bajo estradiol cuando realizaron las pruebas con bajos niveles de estradiol (78-400 pg/ml).

Las puntuaciones directas del inventario IDER fueron pasadas a percentiles, según los baremos de la prueba para mujeres universitarias. Los ítems de eutimia fueron puntuados en orden descendente y los de distimia en orden ascendente para obtener las escalas estado y rasgo del inventario, como indica el manual, pero para obtener los valores de eutimia fuera de las escalas se utilizaron las puntuaciones directas y el mismo orden que para distimia, esto es, ascendente. Los datos fueron analizados con el software estadístico SPSS 22.0. 
Dado que la significación del estadístico de Kolmogorov-Smirnov fue mayor de 0.05 se asumió que la distribución era normal. Los datos fueron analizados mediante la prueba $T$ de Student para medias de dos muestras emparejadas, así como la prueba chi-cuadrado, tomando como punto de corte la media inter-grupal.

A través de la correlación de Pearson se estudió la posible relación entre las variables estradiol, eutimia estado, distimia estado, atribución semántica y atribución facial.

Se utilizó el análisis de varianza unifactorial para evaluar el efecto individual de las variables afectividad (distimia y eutimia) sobre el tipo de atribución realizada tanto a los estímulos semánticos como faciales. Mediante el ANOVA de medidas repetidas se estudió el efecto individual de la variable eutimia y el efecto conjunto de las variables estradiol y eutimia en un diseño (2x3). Para ello se halló la media de las puntuaciones obtenidas en la variable eutimia y se obtuvieron 3 grupos: "eutimia alta" cuyas puntuaciones se encontraban dos desviaciones típicas por encima de la media; "eutimia media" cuyas puntuaciones se encontraban entre dos desviaciones típicas por encima y por debajo de la media; y "eutimia baja" cuyas puntuaciones se encontraban dos desviaciones típicas por debajo de la media. La variable estradiol tenía dos niveles: alto y bajo estradiol. Como el nivel crítico asociado al estadístico $W$ de Mauchly fue mayor que 0.05 , se aceptó el supuesto de esferidad y se optó por la aproximación de los estadísticos $F$ univariados asociados a cada efecto. Todas las pruebas estadísticas se realizaron utilizando un nivel de significación de $p<0.05$.

\section{RESULTADOS}

Las muestras de saliva mostraron valores de 480-940 pg/ml en el día del pico preovulatorio con una media de $672.375 \mathrm{pg} / \mathrm{ml}$; durante la fase lútea la concentración fue menor, de 78-400 pg/ml, con una media de $229.15 \mathrm{pg} / \mathrm{ml}$.

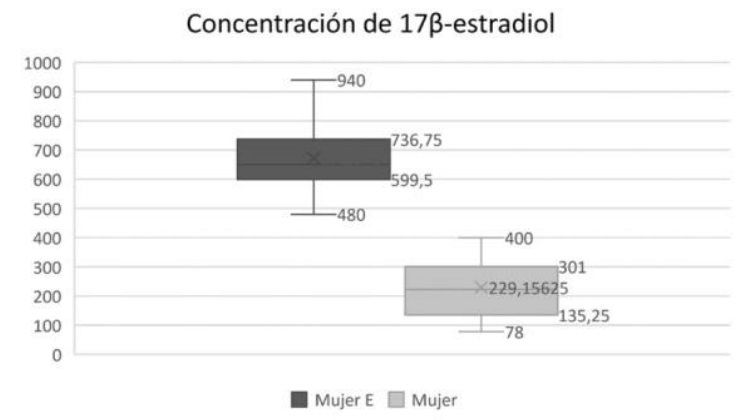

Gráfico 1. Valores de estradiol durante el día del pico preovulatorio (Mujer E) y durante la fase perimenstrual (Mujer) en $\mathrm{pg} / \mathrm{ml}$ 
A través de la prueba de diferencias emparejadas se encontró una diferencia significativa entre la puntuación obtenida en la escala Estado del inventario IDER cuando los niveles de estradiol fueron bajos con respecto al momento en que éstos fueron altos, $t(63)=11.99 ; p=.000$ (bilateral), esta diferencia fue debida a las puntuaciones correspondientes a los ítems de eutimia estado, $t(63)=-15.78 ; p=.000$ (bilateral), no hallándose diferencias significativas en distimia estado ni en la escala rasgo (ver Tabla 1).

Tabla 1. Prueba de muestras emparejadas: Afectividad-Estradiol

\begin{tabular}{|c|c|c|c|c|c|c|c|c|c|}
\hline & & $M$ & $D S$ & $\begin{array}{c}\text { Desv. } \\
\text { Error } \\
\text { promedio }\end{array}$ & $\begin{array}{l}95 \% \text { IC } \\
\text { inferior }\end{array}$ & $\begin{array}{l}95 \% \text { IC } \\
\text { superior }\end{array}$ & $t$ & $\mathrm{gk}$ & $\begin{array}{c}\text { Sig. } \\
\text { (bilateral) }\end{array}$ \\
\hline Par 1 & $\begin{array}{l}\text { A.E. Distimia.Estado } \\
\text { B.E. Distimia.Estado }\end{array}$ & -.0312 & 1.596 & . 282 & -.606 & .544 & -.111 & 31 & .913 \\
\hline Par 2 & $\begin{array}{l}\text { A.E. Eutimia.Estado } \\
\text { B.E. Eutimia.Estado }\end{array}$ & -5.875 & 2.106 & .372 & -6.634 & -5.115 & -15.780 & 31 & .000 \\
\hline Par 3 & $\begin{array}{l}\text { A.E. Estado.Total } \\
\text { B.E. Estado.Total }\end{array}$ & -2.953 & 1.393 & .246 & -3.455 & -2.450 & 11.990 & 31 & .000 \\
\hline Par 4 & $\begin{array}{l}\text { A.E. Distimia.Rasgo } \\
\text { B.E. Distimia.Rasgo }\end{array}$ & -.406 & 1.160 & .205 & -.824 & .012 & -1.981 & 31 & .057 \\
\hline Par 5 & $\begin{array}{l}\text { A.E. Eutimia.Rasgo } \\
\text { B.E. Eutimia.Rasgo }\end{array}$ & -.562 & 1.848 & .326 & -1.228 & .103 & -1.722 & 31 & .095 \\
\hline Par 6 & $\begin{array}{l}\text { A.E. Rasgo.Total } \\
\text { B.E. Rasgo.Total }\end{array}$ & .359 & 1.002 & .177 & -.720 & .001 & -2.029 & 31 & .051 \\
\hline
\end{tabular}

El $90.6 \%$ de los sujetos obtuvieron puntuaciones por encima de la media en los ítems de eutimia estado $\chi^{2}(1, \mathrm{~N}=64)=45.607 ; p=0.00$, frente al $6.3 \%$ cuando sus niveles de estradiol fueron bajos.

La media de las atribuciones de valencia positiva realizadas a los estímulos semánticos fue significativamente mayor $t(63)=-3.66$; $p=.001$ (bilateral) en el grupo Alto estradiol que en el grupo Bajo estradiol, no existiendo diferencias significativas en cuanto al número de atribuciones negativas o neutras.

El porcentaje de sujetos que realizó atribuciones positivas a los estímulos semánticos también fue significativamente mayor $\chi^{2}(1, \mathrm{~N}=64)=9.057 ; p=0.003$, cuando el nivel de estradiol fue elevado (ver Gráfico 2). 


\section{Atribuciones Semánticas}

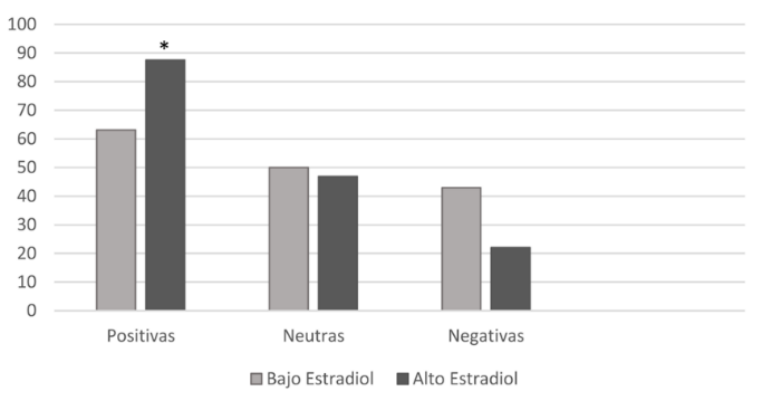

Gráfico 2. Porcentaje de sujetos que hicieron atribuciones positivas, negativas y neutras por encima de la media inter-grupal a una serie de estímulos semánticos. El porcentaje de sujetos que hicieron atribuciones emocionales positivas fue significativamente superior cuando sus niveles de estradiol fueron elevados $(* p=0.003)$, con respecto al momento en que éstos fueron bajos

La media de las atribuciones de valencia positiva realizadas a los estímulos faciales fue significativamente mayor $t(63)=-8.395 ; p=.000$ (bilateral), mientras que la media de atribuciones negativas fue significativamente menor $t(63)=2.784 ; p=.009$ (bilateral), en el grupo Alto estradiol con respecto al grupo Bajo estradiol, así como el porcentaje de sujetos que hizo este tipo de atribuciones, positivas: $\chi^{2}(1, \mathrm{~N}=64)=36.141$; $p=0.00$ y negativas $\chi^{2}(1, \mathrm{~N}=64)=10.827 ; p=0.001$ (ver Gráfico 3$)$.

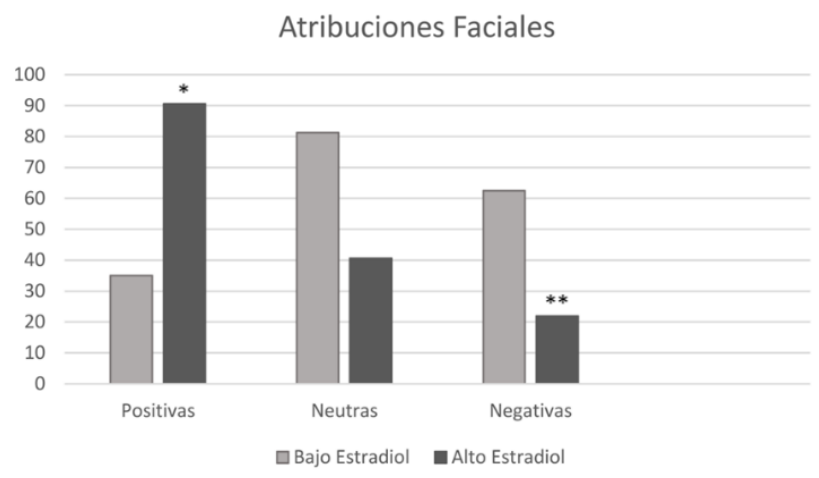

Gráfico 3. Porcentaje de sujetos que hicieron atribuciones positivas, negativas y neutras por encima de la media inter-grupal a una serie de estímulos faciales. El porcentaje de sujetos que hicieron atribuciones emocionales positivas fue significativamente superior cuando sus niveles de estradiol fueron elevados $(* p=0.00)$, con respecto al momento en que éstos fueron bajos. El porcentaje de sujetos que hicieron atribuciones faciales negativas fue significativamente inferior cuando sus niveles de estradiol fueron elevados $(* * p=0.001)$, con respecto al momento en el que fueron bajos 
El tiempo empleado en hacer estas atribuciones a los estímulos semánticos fue significativamente menor en el grupo Alto estradiol, frente al grupo Bajo estradiol, tanto en el tipo de atribuciones positivas $t(63)=2.7 ; p=.011$ (bilateral) como negativas $t(63)=3.373 ; p=.002$ (bilateral), no hubo diferencias significativas en cuanto al tiempo de reacción en el caso de los estímulos faciales.

Existe una correlación positiva muy alta entre el nivel de estradiol y el estado de eutimia. Así mismo, existe una correlación positiva entre el nivel de estradiol y el número de atribuciones de valencia positiva, realizada tanto a los estímulos semánticos como faciales, siendo en este último caso muy alta, y una correlación negativa entre el nivel de estradiol y el número de atribuciones neutras a los estímulos semánticos y faciales y negativas a los estímulos faciales. Puntuaciones similares se encuentran respecto a la variable eutimia y tipo de atribución realizada (ver Tabla 2).

Tabla 2. Correlaciones entre Estradiol-Eutimia-Distimia-Atribuciones Semánticas-Atribuciones Faciales

\begin{tabular}{|c|c|c|c|c|c|c|c|c|c|c|}
\hline & & 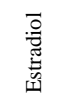 & 氶 & 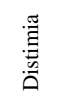 & 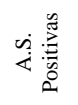 & 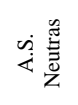 & 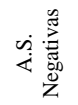 & 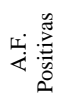 & 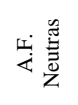 & 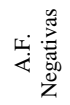 \\
\hline \multirow{3}{*}{ Estradiol } & $\begin{array}{l}\text { Corr. de } \\
\text { Pearson }\end{array}$ & 1 & $.844^{* *}$ & -.191 & $.376^{* *}$ & -.031 & -.219 & $.751^{* *}$ & $-.416^{* *}$ & $-.411^{* *}$ \\
\hline & $\begin{array}{c}\text { Sig. } \\
\text { (bilateral) }\end{array}$ & & .000 & .131 & .002 & .806 & .082 & .000 & .001 & .001 \\
\hline & $\mathrm{N}$ & 64 & 64 & 64 & 64 & 64 & 64 & 64 & 64 & 64 \\
\hline \multirow{3}{*}{ Eutimia } & $\begin{array}{l}\text { Corr. de } \\
\text { Pearson }\end{array}$ & $.844^{* * *}$ & 1 & -.217 & $.356^{* *}$ & -.064 & -.064 & $.597^{* *}$ & $-.313^{*}$ & $-.258^{*}$ \\
\hline & $\begin{array}{c}\text { Sig. } \\
\text { (bilateral) }\end{array}$ & .000 & & .085 & .004 & .618 & .618 & .000 & .012 & .039 \\
\hline & $\mathrm{N}$ & 64 & 64 & 64 & 64 & 64 & 64 & 64 & 64 & 64 \\
\hline \multirow{3}{*}{ Distimia } & $\begin{array}{l}\text { Corr. de } \\
\text { Pearson }\end{array}$ & -.191 & -.217 & 1 & -.120 & -.026 & .101 & -.203 & .055 & .127 \\
\hline & $\begin{array}{c}\text { Sig. } \\
\text { (bilateral) }\end{array}$ & .131 & .085 & & .346 & .839 & .425 & .107 & .666 & .318 \\
\hline & $\mathrm{N}$ & 64 & 64 & 64 & 64 & 64 & 64 & 64 & 64 & 64 \\
\hline \multirow{3}{*}{$\begin{array}{c}\text { A.S. } \\
\text { Positivas }\end{array}$} & $\begin{array}{l}\text { Corr. de } \\
\text { Pearson }\end{array}$ & $.376^{* *}$ & $.356^{* *}$ & -.120 & 1 & $-.465^{* *}$ & .082 & .212 & -.030 & -.137 \\
\hline & $\begin{array}{c}\text { Sig. } \\
\text { (bilateral) }\end{array}$ & .002 & .004 & .346 & & .000 & .518 & .093 & .817 & .279 \\
\hline & $\mathrm{N}$ & 64 & 64 & 64 & 64 & 64 & 64 & 64 & 64 & 64 \\
\hline \multirow{3}{*}{$\begin{array}{c}\text { A.S. } \\
\text { Neutras }\end{array}$} & $\begin{array}{l}\text { Corr. de } \\
\text { Pearson }\end{array}$ & -.031 & -.064 & -.026 & $-.465^{* *}$ & 1 & $-.627^{* *}$ & -.092 & -.057 & -.005 \\
\hline & $\begin{array}{c}\text { Sig. } \\
\text { (bilateral) }\end{array}$ & .806 & .618 & .839 & .000 & & .000 & .470 & .654 & .969 \\
\hline & $\mathrm{N}$ & 64 & 64 & 64 & 64 & 64 & 64 & 64 & 64 & 64 \\
\hline \multirow{3}{*}{$\begin{array}{c}\text { A.S. } \\
\text { Negativas }\end{array}$} & $\begin{array}{l}\text { Corr. de } \\
\text { Pearson }\end{array}$ & -.219 & -.064 & .101 & .082 & $-.627^{* * *}$ & 1 & -.092 & .135 & .122 \\
\hline & $\begin{array}{c}\text { Sig. } \\
\text { (bilateral) }\end{array}$ & .082 & .618 & .425 & .518 & .000 & & .470 & .287 & .338 \\
\hline & $\mathrm{N}$ & 64 & 64 & 64 & 64 & 64 & 64 & 64 & 64 & 64 \\
\hline \multirow{3}{*}{$\begin{array}{c}\text { A.F. } \\
\text { Positivas }\end{array}$} & $\begin{array}{l}\text { Corr. de } \\
\text { Pearson }\end{array}$ & $.751^{* *}$ & $.597^{* *}$ & -.203 & .212 & -.092 & -.092 & 1 & $-.560^{* *}$ & $-.275^{*}$ \\
\hline & $\begin{array}{c}\text { Sig. } \\
\text { (bilateral) }\end{array}$ & .000 & .000 & .107 & .093 & .470 & .470 & & .000 & .028 \\
\hline & $\mathrm{N}$ & 64 & 64 & 64 & 64 & 64 & 64 & 64 & 64 & 64 \\
\hline
\end{tabular}


Tabla 2(continuación). Correlaciones entre Estradiol-Eutimia-Distimia-Atribuciones Semánticas-Atribuciones Faciales

\begin{tabular}{|c|c|c|c|c|c|c|c|c|c|c|}
\hline \multirow{3}{*}{$\begin{array}{c}\text { A.F. } \\
\text { Neutras }\end{array}$} & $\begin{array}{l}\text { Corr. de } \\
\text { Pearson }\end{array}$ & $-.416^{* *}$ & $-.313^{*}$ & .055 & -.030 & -.057 & .135 & $-.560^{* * *}$ & 1 & -.159 \\
\hline & $\begin{array}{c}\text { Sig. } \\
\text { (bilateral) }\end{array}$ & .001 & .012 & .666 & .817 & .654 & .287 & .000 & & .209 \\
\hline & $\mathrm{N}$ & 64 & 64 & 64 & 64 & 64 & 64 & 64 & 64 & 64 \\
\hline \multirow{3}{*}{$\begin{array}{c}\text { A.F. } \\
\text { Negativas }\end{array}$} & $\begin{array}{l}\text { Corr. de } \\
\text { Pearson }\end{array}$ &,$- 411^{* *}$ &,$- 258^{*}$ &, 127 &,- 137 &,- 005 &, 122 &,$- 275^{*}$ &,- 159 & 1 \\
\hline & $\begin{array}{c}\text { Sig. } \\
\text { (bilateral) }\end{array}$ & ,001 & 039 & ,318 &, 279 & ,969 & ,338 & ,028 & ,209 & \\
\hline & $\mathrm{N}$ & 64 & 64 & 64 & 64 & 64 & 64 & 64 & 64 & 64 \\
\hline
\end{tabular}

A través del ANOVA unifactorial no se encontró ningún efecto significativo del factor Distimia sobre las variables atribución semántica y facial. Sin embargo, se encontró un efecto significativo del factor Eutimia y las variables atribuciones semánticas positivas $(F=9.001 ; p=0.004)$ y atribuciones faciales positivas $(F=4.428$; $p=0.039$ ).

Los resultados del ANOVA con medidas repetidas cuando se comparan el factor Eutimia (alto/medio/bajo) y el factor estradiol (alto/bajo) sobre el tipo de atribución realizada a los estímulos semánticos mostraron un efecto significativo del primer factor, nivel de estradiol $(F=7.846 ; p=0.019)$, y del segundo factor, Eutimia, $(F=7.285 ; p=0.004)$ sólo en el tipo de atribución de valencia positiva, un efecto similar se encontró cuando se estudia el efecto del factor estradiol sobre el tipo de atribución realizada a los estímulos faciales $(F=10.126 ; p=0.024)$, mientras que el factor Eutimia mostró un efecto significativo en el número de atribuciones faciales positivas y negativas ( $\mathrm{F}=11.591 ; p=0.007$ y $F=13.144 ; p=0.005$, respectivamente), la interacción de los factores estradiol-eutimia sobre el tipo de atribución semántica $(F=10.126 ; p=0.024) \mathrm{y}$ facial $(F=7.945 ; p=0.003)$, mostró resultados semejantes, sólo con respecto a las atribuciones de valencia positiva.

\section{DISCUSIÓN Y CONCLUSIONES}

La fase lútea tardía y particularmente el inicio del periodo premenstrual (momentos en el que los niveles de estradiol son bajos) están asociados en muchas mujeres con síntomas psicológicos como un incremento de la depresión, ansiedad, irritabilidad o tensión y cambios de humor, síntomas que disminuyen hacia el momento de la ovulación (momento en el que los niveles de estradiol son altos) para aumentar nuevamente en la fase premenstrual, mostrando una distribución curvilínea en forma de U a lo largo del ciclo, (Lorenz, Gesselman, y Vitzthum, 2017; Sundström-Poromaa, 2018).

Si bien en este estudio se observa una ligera disminución de los síntomas de distimia cuando los niveles de estradiol son elevados, los resultados muestran que la 
mejoría en el estado de ánimo en una población no clínica puede deberse más al incremento de síntomas afectivos positivos que a la disminución de los negativos, como han sugerido otras investigaciones (Bayer, Schultz, Gamer, y Sommer, 2014; Gordon et al., 2018; Li y Graham, 2017).

Los resultados de este experimento muestran una mayor tendencia a valorar como más positivos, y menos negativos, los estímulos faciales cuando los niveles de estradiol son más elevados. El estradiol ha sido asociado tanto a una mejoría en el procesamiento de las señales sociales (Marečková et al., 2014) como a la regulación de la memoria social (Karlsson et al., 2016) y parece intervenir en la respuesta hacia los acontecimientos psicosocialmente estresantes, la cual precede a menudo a los episodios depresivos y ansiosos, atenuando el distrés subjetivo, el nivel de cortisol, los efectos negativos del estrés en la memoria de trabajo y la respuesta de humor negativa, y actuando como un factor de resiliencia ante estas situaciones (Albert, Pruessner, y Newhouse, 2015; Herrera, Hodis, Mack, Mather, y Mather, 2017).

El estado de ánimo, o afectivo, puede dar un tono positivo o negativo a la experiencia a través de atajos cognitivos, o heurísticos, que nos permiten simplificar el proceso de razonamiento, convirtiendo en congruente cómo nos sentimos con lo que sentimos hacia nuestro entorno, los juicios y las actitudes que elaboramos. De esta forma, el estado afectivo actúa como una clave de recuperación que facilita la evocación posterior de los eventos vividos (Grupe et al., 2018).

Los sujetos de esta investigación mostraron una mayor disposición a valorar favorablemente los estímulos presentados, tanto semánticos como faciales, cuando sus niveles de estradiol fueron elevados que cuando éstos fueron bajos. Un mayor uso de atribuciones positivas, y un menor uso de las negativas, en la autopercepción, la interpretación, la atención, y la memoria pueden suponer un factor de prevención ante el riesgo de padecer depresión (Preece, Becerra, Robinson, Dandy, y Allan, 2018). Algunos autores sostienen que es la tendencia a interpretar los estímulos como más negativos, o menos positivos, de lo que realmente son, es lo que determina el mayor riesgo a padecer depresión (Brockmeyer, Kulessa, Hautzinger, Bent, y Backenstrass, 2015; Meilán, Carro, Guerrero, Carpi, Gómez, y Palmero, 2012). En mujeres con trastorno disfórico premenstrual los errores en la identificación de la emoción de expresiones faciales son congruentes con su humor y similares a los que se manifiestan en los trastornos de depresión, esto es, presentan una tendencia negativa en sus atribuciones emocionales (ven las caras más tristes de lo que son), esta tendencia, junto con el déficit que manifiestan en la discriminación emocional, aumenta durante la fase lútea, lo que puede contribuir a la generación de estados de humor negativos (Rubinow el al., 2007).

Los resultados de esta investigación proponen que sería durante la fase folicular del ciclo menstrual donde el empleo y el entrenamiento de estrategias de control y regulación emocional resultaría más eficaz, permitiendo adaptar de una manera 
más positiva, y congruente con el estado de ánimo, el material cognoscitivo y ayudando a evitar algunos de los sesgos negativos propios de la depresión. El estado afectivo positivo afecta a la evaluación de estímulos neutros, siendo calificados como más agradables y favoreciendo la motivación, la atención y la memoria a largo plazo (Grupe et al., 2018), algunos autores han hallado una asociación entre este sesgo positivo y la activación de la amígdala y la corteza prefrontal, por un incremento de los niveles sinápticos de DA, durante la fase folicular (Mulligan et al., 2018).

Los datos obtenidos en este estudio permiten comprobar que el fenómeno de respuestas congruentes con el estado de ánimo ocurre también en el estado afectivo positivo, y no sólo en el estado afectivo negativo, que ha sido la dimensión más estudiada de forma sistemática, lo que sugiere que el estradiol podría estar implicado en la generación de experiencias y expectativas positivas, y en la reducción de las negativas (da Costa y Páez, 2015) pudiendo favorecer la construcción de recursos personales para el desarrollo y la protección a nivel físico, emocional, social e intelectual.

\section{REFERENCIAS}

Albert, K., Pruessner, J. y Newhouse, P. (2015). Estradiol Levels Modulate Brain Activity and Negative Responses to Psychosocial Stress across the Menstrual Cycle. Psychoneuroendocrinology, 59, 14-24.

Armbruster, D., Grage, T., Kirschbaum, C. y Strobel, A. (2018). Processing emotions: Effects of menstrual cycle phase and premenstrual symptoms on the startle reflex, facial EMG and heart rate. Behav Brain Res. 1(351), 178-187.

Bayer, J., Schultz, H., Gamer, M. y Sommer, T. (2014). Menstrual-cycle dependent fluctuations in ovarian hormones affect emotional memory. Neurobiology of Learning and Memory, $110,55-63$.

Brockmeyer, T., Kulessa, D., Hautzinger, M., Bents, H. y Backenstrass, M. (2015). Moodincongruent processing during the recall of a sad life event predicts the course and severity of depression. Journal of Affective Disorders, 187, 91-96.

Bush, K.A., Inman, C.S., Hamann, S., Kilts, C.D. y James, G.A. (2017). Distributed Neural Processing Predictors of Multi-dimensional Properties of Affect. Frontiers in Human Neuroscience, $11,459$.

da Costa, S. y Páez, D. (2015). Afectividad inducida e impacto en la creatividad, crecimiento personal después del cambio y ajuste percibido al narrar una experiencia emocional intensa. Anales de Psicología, 31(2), 716-724.

Del Río, J.P., Alliende, M.I., Molina, N., Serrano, F.G., Molina, S. y Vigil, P. (2018). Steroid Hormones and Their Action in Women's Brains: The Importance of Hormonal Balance. Frontiers in Public Health, 6, 141.

Diekhof, E.K. y Ratnayake, M. (2016). Menstrual cycle phase modulates reward sensitivity and performance monitoring in young women: Preliminary fMRI evidence. Neuropsychologia, 84, 70-80.

Dreher, J.C., Schmidt, P.J., Kohn, P., Furman, D., Rubinow, D. y Berman, K.F. (2007). Menstrual cycle phase modulates reward-related neural function in women. Proceedings of the National Academy of Sciences of the United States of America, 104(7), 2465-2470. 
Fernández, A., Díez, E., Alonso, M.A. y Beato, M.S. (2004). Free-association norms for the Spanish names of the Snodgrass and Vanderwart pictures. Behavior Research Methods, Instruments, \& Computers, 36, 577-583.

Flöter A.J., Nathorst-Böös, J., Carlström, K. y von Schoultz, B. (2002). Addition of testosterone to estrogen replacement therapy in oophorectomized women: effects on sexuality and wellbeing, Climacteric, 5(4), 357-365.

Gordon, J.L., Rubinow, D.R., Eisenlohr-Moul, T.A., Xia, K., Schmidt, P.J. y Girdler, S.S. (2018). Efficacy of Transdermal Estradiol and Micronized Progesterone in the Prevention of Depressive Symptoms in the Menopause TransitionA Randomized Clinical Trial. JAMA Psychiatry, 75(2), 149-157.

Graham, B.M., Denson, T.F., Barnett, J., Calderwood, C. y Grisham, J.R. (2018). Sex Hormones Are Associated With Rumination and Interact With Emotion Regulation Strategy Choice to Predict Negative Affect in Women Following a Sad Mood Induction. Frontiers in Psychology, 9, 937.

Grupe, D.W., Schaefer, S.M., Lapate, R.C., Schoen, A.J., Gresham, L.K., Mumford, J.A. y Davidson, R.J. (2018). Behavioral and neural indices of affective coloring for neutral social stimuli. Social Cognitive and Affective Neuroscience, 13(3), 310-320.

Herrera, A.Y., Hodis, H.N., Mack, W.J. y Mather, M. (2017) Estradiol Therapy After Menopause Mitigates Effects of Stress on Cortisol and Working Memory. The Journal of Clinical Endocrinology y Metabolism. J Clin Endocrinol, 102(12),4457-4466.

Karlsson, S., Henningsson, S., Hovey, D., Zettergren, A., Jonsson, L., Cortes, D.S. y Westberg, L. (2016). Social memory associated with estrogen receptor polymorphisms in women. Social Cognitive and Affective Neuroscience, 11(6), 877-883.

Kiesner, J., Mendle, J., Eisenlohr-Moul, T.A. y Pastore, M. (2016). Cyclical symptom change across the menstrual cycle. Clin. Psychol. Sci. 4, 882-894.

Le Moult, J. y Gotlib, I.H. (2018). Depression: A cognitive perspective. Clinical Psychology Review, (17), 30391-30394.

Li, S.H. y Graham, B.M. (2017). Why are women so vulnerable to anxiety, trauma- and stressrelated disorders? The potential role of sex hormones. Lancet Psychiatry, 4,73-82.

Lorenz, T.K., Gesselman, A.N. y Vitzthum, V.J. (2017). Variance in Mood Symptoms Across Menstrual Cycles: Implications for Premenstrual Dysphoric Disorder. Women's Reproductive Health (Philadelphia, Pa.), 4(2), 77-88.

Marečková, K., Perrin, J.S., Nawaz Khan, I., Lawrence, C., Dickie, E. y McQuiggan, D.A. (2014). Hormonal contraceptives, menstrual cycle and brain response to faces. Social Cognitive and Affective Neuroscience, 9(2), 191-200.

Mulligan, E.M., Nelson, B.D., Infantolino, Z.P., Luking, K.R., Sharma, R. y Hajcak, G. (2018). Effects of menstrual cycle phase on electrocortical response to reward and depressive symptoms in women. Psychophysiology, 16 PÁG

Natale, V., Albertazzi, P. y Cangini, A. (2003). The Effects of Menstrual Cycle on Dreaming. Biological Rhythm Research, 34(3), 295-303.

Okely, J.A., Weiss, A. y Gale, C.R. (2017). The interaction between stress and positive affect in predicting mortality. Journal of Psychosomatic Research, 100, 53-60.

Pletzer, B., Kronbichler, M., Aichhorn, M., Bergmann, J., Ladurner, G. y Kerschbaum, H.H. (2010). Menstrual cycle and hormonal contraceptive use modulate human brain structure. Brain Res. 12(1348), 55-62.

Preece, D., Becerra, R., Robinson, K., Dandy, J. y Allan, A. (2018). Measuring emotion regulation ability across negative and positive emotions: The Perth Emotion Regulation Competency Inventory (PERCI). Personality and Individual Differences, 135, 229-241. 
Rubinow, D. R., Smith, M.J., Schenkel, L.A., Schmidt, P.J. y Dancer, K. (2007). Facial Emotion Discrimination Across the Menstrual Cycle in Women with Premenstrual Dysphoric Disorder (PMDD) and Controls. Journal of Affective Disorders, 104(1-3), 37-44.

Schiller, D. y Delgado, M.R. (2010). Overlapping neural systems mediating extinction, reversal and regulation of fear. Trends Cogn. Sci. 14, 268-276.

Schwarz, J., Rief, W., Radkovsky, A., Berking, M. y Kleinstauber, M. (2017). Negative affect as mediator between emotion regulation and medically unexplained symptoms. Journal of Psychosomatic Research, 101,114-121.

Sundström-Poromaa (2018). The Menstrual Cycle Influences Emotion but Has Limited Effect on Cognitive Function. Vitam Horm. 107, 349-376.

Spielberger, C.D., Agudelo, D. y Buela-Casal, G. (2008). Inventario de Depresión Estado/Rasgo (IDER). Madrid: TEA Ediciones.

Spielberger, C.D., Ritterband, L., Reheiser, E. y Brunner, T. (2003). The nature and measurement of depression. Revista Internacional de Psicología Clínica y de la Salud/International Journal of Clinical and Health Psychology, 3, 209-234. Recuperado de http://www.redalyc.org/pdf/337/33730201.pdf

Spindler, H., Denollet, J., Kruse, C. y Pedersen, S.S. (2009). Positive affect and negative affect correlate differently with distress and health-related quality of life in patients with cardiac conditions: Validation of the Danish Global Mood Scale. Journal of Psychosomatic Research, 67(1), 57-65.

van Wingen, G., van Broekhoven, F., Verkes R.J., Petersson, K.M., Bäckström, T. y Buitelaar, J. (2008). Progesterone selectively increases amygdala reactivity in women. Mol. Psychiatry, VOL/NUM, 13325-13333.

Wang, X., Wang, Y., Ma, L., Zhang, R., De, Y., Yang, X. y Wu, Q. (2015). Development of an improved competitive ELISA based on a monoclonal antibody against lipopolysaccharide for the detection of bovine brucellosis. BMC Veterinary Research, $11,118$.

Yen, J.Y., Wang, P.W., Su, C.H., Liu, T.L., Long, C.Y. y Ko, C.H. (2018). Estrogen levels, emotion regulation, and emotional symptoms of women with premenstrual dysphoric disorder: The moderating effect of estrogen receptor $1 \alpha$ polymorphism. Prog Neuropsychopharmacol Biol Psychiatry. 2(82), 216-223.

Young, A., Perrett, D.I., Calder, A., Sprengelmeyer, R.H. y Ekman, P.(2002). Facial expressions of emotion: Stimuli and Test (FEEST).

Zhang, Y. y Han, B. (2016). Positive affect and mortality risk in older adults: A meta-analysis. Psych J. Jun, 5(2):125-138.

Recibido: 07 de octubre de 2018 Recepción Modificaciones: 21 de octubre de 2018

Aceptado: 25 de octubre de 2018 